\title{
Longterm Outcomes of 188 Japanese Patients with Eosinophilic Granulomatosis with Polyangiitis
}

\author{
Aiko Saku, Shunsuke Furuta, Masaki Hiraguri, Kei Ikeda, Yoshihisa Kobayashi, \\ Shin-ichiro Kagami, Kazuhiro Kurasawa, Ryutaro Matsumura, Daiki Nakagomi, \\ Takao Sugiyama, Takeshi Umibe, Norihiko Watanabe, and Hiroshi Nakajima
}

ABSTRACT. Objective. Patients with eosinophilic granulomatosis with polyangiitis (EGPA) frequently experience relapses, which lead to cumulative organ damage. In this retrospective observational study, we aimed to reveal the risk factors for relapse in EGPA.

Methods. A total of 188 Japanese patients with EGPA diagnosed between 1996 and 2015 were identified from medical records in 10 hospitals. The diagnosis was based on the American College of Rheumatology 1990 criteria or Lanham's criteria. Baseline characteristics, treatments, asthma exacerbation, and relapses were evaluated by retrospective chart review.

Results. The median followup period was 56 months. The median age at disease onset was 59.7 years. At the disease onset, $95.2 \%$ of the patients had a history of bronchial asthma and $44.7 \%$ were positive for antineutrophil cytoplasmic antibodies. The cumulative survival and relapse-free survival rates at 5 years were $89.6 \%$ and $64.0 \%$, respectively. Multivariate analysis with 2 models, proportional hazards, and competing risk models, was performed to identify the factors associated with relapse. The proportional hazards model identified azathioprine (AZA) maintenance therapy and high eosinophil counts at onset as independent factors with lower relapse risks, and high immunoglobulin $\mathrm{E}$ (IgE) levels at onset as a risk factor for relapse. The competing risk model identified no statistically significant factors.

Conclusion. Although potential benefit of AZA maintenance therapy in preventing relapse of EGPA was suggested by the proportional hazards model, there was a discrepancy in the results between the models. Eosinophil counts and IgE levels at onset were also identified as candidates of factors associated with relapse in EGPA. (First Release June 15 2018; J Rheumatol 2018;45:1159-66; doi:10.3899/jrheum.171352)

Key Indexing Terms:

ANTINEUTROPHIL CYTOPLASMIC ANTIBODIES-ASSOCIATED VASCULITIS

EOSINOPHILIC GRANULOMATOSIS WITH POLYANGIITIS OUTCOME RELAPSE JAPAN

From the Department of Allergy and Clinical Immunology, Chiba

University Hospital; Department of Internal Medicine, Narita Red Cross

Hospital; Department of Internal Medicine, Chiba Aoba Municipal

Hospital; Department of Allergy and Clinical Immunology, Asahi General

Hospital, Chiba; Department of Rheumatology, Dokkyo Medical

University, Tochigi; Department of Rheumatology, Allergy and Clinical

Immunology, National Hospital Organization Chiba-East Hospital, Chiba; Third Department of Internal Medicine, University of Yamanashi,

Yamanashi; Department of Rheumatology, National Hospital Organization Shimoshizu Hospital; Department of Internal Medicine, Matsudo City

Hospital; Centre for Rheumatic Diseases, Chibaken Saiseikai Narashino Hospital, Chiba, Japan.

A. Saku, MD, Chiba University Hospital; S. Furuta, MD, PhD, Chiba University Hospital; M. Hiraguri, MD, PhD, Narita Red Cross Hospital; K. Ikeda, MD, PhD, Chiba University Hospital; Y. Kobayashi, MD, PhD, Chiba Aoba Municipal Hospital; S.I. Kagami, MD, PhD, Asahi General Hospital; K. Kurasawa, MD, PhD, Dokkyo Medical University;

R. Matsumura, MD, PhD, Chiba-East Hospital; D. Nakagomi, MD, PhD,

University of Yamanashi; T. Sugiyama, MD, PhD, Shimoshizu Hospital;

T. Umibe, MD, PhD, Matsudo City Hospital; N. Watanabe, MD, PhD,

Chibaken Saiseikai Narashino Hospital; H. Nakajima, MD, PhD, Chiba University Hospital.

Address correspondence to Dr. S. Furuta, MD, PhD, Department of

Allergy and Clinical Immunology, Chiba University Hospital,

1-8-1 Inohana, Chuo-ku, Chiba-shi, Chiba 260-8670, Japan.

E-mail: shfuruta@chiba-u.jp

Accepted for publication March 7, 2018.
Eosinophilic granulomatosis with polyangiitis (EGPA, formerly Churg-Strauss syndrome) is a rare form of antineutrophil cytoplasmic antibody (ANCA)-associated vasculitides (AAV). It is characterized by eosinophil-rich and necrotizing granulomatous inflammation, necrotizing vasculitis predominantly affecting small to medium vessels, and association with asthma and eosinophilia ${ }^{1,2}$. It has been reported that prevalence and annual incidence of EGPA in Japan are 17.8 per million adults and 2.4 per million adults, respectively, similar to those in Western countries ${ }^{3,4}$. EGPA has often been excluded from clinical studies and trials regarding AAV because of its rarity and unique features. Consequently, there is still much uncertainty about the optimal management of EGPA compared to more common forms of AAV, microscopic polyangiitis (MPA), and granulomatosis with polyangiitis (GPA).

Inflammation in EGPA is mainly composed of eosinophil. Glucocorticoids can diminish human eosinophils through direct and indirect pathways ${ }^{5}$. Thus, response to glucocorticoids in EGPA is generally good, and glucocorticoid induction therapy with or without immunosuppressants leads

Personal non-commercial use only. The Journal of Rheumatology Copyright @ 2018. All rights reserved. 
to successful remission in most patients with $\mathrm{EGPA}^{6,7}$. However, it has been well-known that these patients experience frequent relapses during glucocorticoid tapering $8,9,10,11$. It has been reported that the number of relapses is associated with cumulative organ damage in patients with $\mathrm{AAV}^{12}$. Therefore, to know factors associated with relapse and to prevent relapses are crucial for the management of EGPA.

Factors associated with mortality in patients with EGPA have been studied. In 1996, the French Vasculitis Study Group revealed that proteinuria, elevated serum creatinine level, cardiomyopathy, gastrointestinal (GI) manifestations, and central nervous system involvement were risk factors for premature death and developed the 5-factor score (FFS) as a predictor by using a large mixed cohort of polyarteritis nodosa, MPA, and EGPA ${ }^{13}$. In 2011, they revisited FFS by using a cohort including GPA, and now the FFS consists of age $>65$ years, cardiac and GI symptoms, elevated serum creatinine levels, and the absence of ear, nose, and throat symptoms ${ }^{14}$. Trials suggested that therapeutic strategies of immunosuppressant use in remission induction phase according to FFS improves mortality in patients with EGPA $^{7,15}$. Higher age and cardiac involvement were also identified as risk factors for death in other cohort studies with

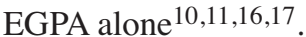

Regarding risk factors for relapse, on the other hand, few retrospective observational studies have been conducted, and their results were inconsistent ${ }^{9,11,16,17}$. At the moment, there is still much uncertainty in risk factors for relapse in EGPA. In addition, immunosuppressant use as remission maintenance therapy is of uncertain value in EGPA, while it has been established already in other forms of AAV such as MPA and GPA ${ }^{18,19}$.

In this retrospective observational study, we aimed to investigate factors associated with relapse in patients with EGPA.

\section{MATERIALS AND METHODS}

Patients. A total of 188 Japanese patients with EGPA diagnosed between October 1996 and October 2015 were identified from medical records in 10 Japanese centers: Chiba University Hospital, National Hospital Organization Shimoshizu Hospital, University of Yamanashi Hospital, Chiba Aoba Municipal Hospital, Asahi General Hospital, Dokkyo Medical University, Chibaken Saiseikai Narashino Hospital, Narita Red Cross Hospital, Matsudo City Hospital, and the National Hospital Organization Chiba-East Hospital. The diagnosis of EGPA in our study was based on either the American College of Rheumatology (ACR) 1990 criteria for the classification of Churg-Strauss syndrome ${ }^{20}$ or Lanham's criteria ${ }^{21}$.

Assessment. Baseline characteristics included age, sex, ANCA status, white blood cell count (WBC), eosinophil count, serum immunoglobulin E (IgE), serum creatinine, C-reactive protein (CRP), organ involvement, and FFS. Myeloperoxidase/proteinase 3 (MPO/PR3)-ANCA results were available for almost all patients, but results of indirect immunofluorescence were used when only these data were available. Diagnosis of organ involvement was based on presence of the symptoms listed in Birmingham Vasculitis Activity Score (BVAS) items ${ }^{22,23}$. Organ involvement in our study was not always biopsy-proven.
Also recorded were treatments for EGPA, the number of deaths, time to death, the number of relapses, time to relapse, the number of asthma exacerbations, and time to asthma exacerbation. Regarding the treatments, variables evaluated were the oral glucocorticoid dose converted to the equivalent prednisolone dose at onset, 6, 12, and 36 months; use of methylprednisolone pulse; immunosuppressants; intravenous immunoglobulin (IVIG); biologic agents; and cumulative cyclophosphamide (CYC) exposure.

Remission was defined as the absence of any disease activity related to active vasculitis regardless of treatment intensity. Relapse was defined as recurrence of vasculitis requiring treatment change or increasing dose of glucocorticoids, and/or adding immunosuppressants, biologic agents, or IVIG. Asthma exacerbation was not included in relapse in our study.

Data were retrospectively acquired from patients' medical charts and computer records.

Statistics. The distributions of age, WBC, eosinophil count, serum IgE, serum creatinine, and CRP levels were described by median and range, and compared by Mann-Whitney U test. Proportions of sex, ANCA serotype, and organ involvement were compared by chi-square test, or Fisher's exact test when the expected frequency was $<5$ in 1 or more cells. The cumulative rates for survival and relapse-free survival were assessed by Kaplan-Meier survival curve, and compared by log-rank test. Risk factors for relapse were assessed by univariate and multivariate analysis with a proportional hazards model. In classic survival analysis and multivariate analysis with a proportional hazards model, relapse-free survival is the time until relapse or death, whatever occurs first. A competing risk model allows jointly assessing an effect on the event of interest (relapse but not death) and an effect on death without prior relapse (a competing risk). For more specific results about relapse, the cumulative rates for relapse were also assessed by cumulative incidence function and compared by Gray's test. Similarly, risk factors for relapse were assessed by univariate and multivariate analysis with a competing risk model (Fine and Gray model). All analyses were performed using SAS version 9.3, and $\mathrm{p}<0.05$ was taken to indicate statistical significance.

Ethics. This study was approved by the ethics committee of Chiba University School of Medicine (reference number: 2002). Patient's written informed consent was not obtained because it was not required by local regulations in the case of a retrospective observational study.

\section{RESULTS}

Baseline characteristics. Baseline characteristics are shown in Table 1. The median age at onset was 59.7 years. At diagnosis of EGPA, 179 of 188 patients (95.2\%) had a history of bronchial asthma for median 5.5 years. The proportion of MPO-ANCA-positive patients was $41.9 \%$, while that of PR3-ANCA-positive patients was $5.4 \%$. Eosinophil count was highly elevated in accordance with the ACR or Lanham's criteria (median $8775 / \mu \mathrm{l}$ ). The most frequent organ involvement at diagnosis was nerve system (88.3\%), followed by ear, nose, and throat $(50.5 \%)$ and skin lesions (41.5\%). Kidney involvement was more frequent in ANCA-positive patients than in ANCA-negative patients $(32.1 \%$ vs $6.9 \%$, $\mathrm{p}<0.01$ ), while heart involvement was less frequent in ANCA-positive patients than in ANCA-negative patients (5.9\% vs $15.8 \%, \mathrm{p}=0.03)$, data that were consistent with the previous report ${ }^{17}$. No other difference was found in organ involvement between ANCA- positive and -negative patients.

Treatments. At diagnosis of EGPA, oral glucocorticoids were administered to most patients (98.9\%), with median prednisolone equivalent dose of $40 \mathrm{mg} /$ day (Table 2). Methylprednisolone pulse therapy was also used in 59

Personal non-commercial use only. The Journal of Rheumatology Copyright @ 2018. All rights reserved 
Table 1. Baseline characteristics of the patients with EGPA $(\mathrm{n}=188)$.

\begin{tabular}{|c|c|}
\hline Characteristics & Values \\
\hline Age at onset, yrs (IQR) & $59.7(49.8-70.4)$ \\
\hline Male: Female (female rate) & $67: 121(64.4 \%)$ \\
\hline Presence of asthma, n (\%) & $179(95.2)$ \\
\hline Presence of other allergy, n (\%) & $105(56.4)$ \\
\hline \multicolumn{2}{|l|}{ Duration from asthma onset to EGPA } \\
\hline diagnosis, yrs (IQR) & $5.5(2-14)$ \\
\hline MPO-ANCA-positive, n (\%) & $78(41.9)$ \\
\hline PR3-ANCA-positive, n (\%) & $10(5.4)$ \\
\hline White blood cell count, / $\mu \mathrm{l}$ (IQR) & $18,000(13,400-24,525)$ \\
\hline Eosinophil, / $\mu \mathrm{l}$ (IQR) & $8775(4802-15,876)$ \\
\hline Immunoglobulin E, IU/ml (IQR) & $1029(465-1717)$ \\
\hline C-reactive protein, mg/dl (IQR) & $2.90(1.04-7.60)$ \\
\hline Creatinine, mg/dl (IQR) & $0.68(0.51-0.80)$ \\
\hline \multicolumn{2}{|l|}{ Organ involvement, n (\%) } \\
\hline Nerve & $166(88.3)$ \\
\hline Skin & $78(41.5)$ \\
\hline Eye & $10(5.3)$ \\
\hline Ear, nose, and throat & $95(50.5)$ \\
\hline Respiratory & $65(34.6)$ \\
\hline Heart & $21(11.2)$ \\
\hline Intestine & $23(12.2)$ \\
\hline Kidney & $34(18.1)$ \\
\hline \multicolumn{2}{|l|}{ 5-factor score, n (\%) } \\
\hline 0 & $120(63.8)$ \\
\hline 1 & $60(31.9)$ \\
\hline 2 & $8(4.2)$ \\
\hline Followup period, months (IQR) & $56(21-102)$ \\
\hline
\end{tabular}

Median values are given for age at the onset, duration from asthma onset to EGPA diagnosis, white blood cell count, eosinophil count, serum immunoglobulin E, C-reactive protein, creatinine levels, and followup period. EGPA: eosinophilic granulomatosis with polyangiitis; IQR: interquartile range; MPO: myeloperoxidase; PR3: proteinase 3; ANCA: antineutrophil cytoplasmic antibody.

Table 2. Initial treatments for EGPA. Data are n (\%) unless otherwise indicated.

\begin{tabular}{lc}
\hline Variables & $\mathrm{n}=188$ \\
\hline Oral glucocorticoids & $186(98.9)$ \\
Maximum dose mg/day, median (IQR) & $40(40-60)$ \\
Methylprednisolone pulse therapy & $59(31.4)$ \\
Immunosuppressants & $56(29.8)$ \\
Cyclophosphamide & $56(29.8)$ \\
Azathioprine & $4(2.1)$ \\
Cyclosporine & $3(1.6)$ \\
Intravenous immunoglobulins & $39(20.7)$ \\
Biologic agents & $3(1.6)$
\end{tabular}

EGPA: eosinophilic granulomatosis with polyangiitis; IQR: interquartile range.

patients $(31.4 \%)$. In Japan, IVIG is formally approved for EGPA by the government, and consequently frequent use of IVIG $(20.7 \%)$ was observed in this cohort. The median prednisolone doses at 6,12 , and 36 months were $15 \mathrm{mg} /$ day, $10 \mathrm{mg} / \mathrm{day}$, and $7.5 \mathrm{mg} /$ day, respectively. One hundred thirty patients $(69.1 \%)$ were treated with glucocorticoid mono- therapy, whereas 56 patients $(29.8 \%)$ were treated with a combination of glucocorticoids and immunosuppressants in the remission induction phase ( $<6 \mathrm{mos})$. In the combination therapy group, all 56 patients initially received CYC, with median cumulative dose of $1.2 \mathrm{~g} /$ body. Four of 56 patients switched to azathioprine (AZA) and 3 of 56 patients switched to cyclosporine (CSA) for their refractory diseases during the remission induction phase. After the remission induction phase, 17 of 56 patients continued immunosuppressants as maintenance therapy (14 AZA and 3 CSA). Two patients received rituximab and 1 received omalizumab for their refractory disease.

At the time of the first relapse, median dose of prednisolone was $8.0 \mathrm{mg} /$ day. Four of 52 patients had stopped prednisolone before their relapses. Forty-four of 52 patients increased their dose of prednisolone, and median increased dose was $20 \mathrm{mg} /$ day. Methylprednisolone pulse therapy was also used in 4 patients. In addition, various immunosuppressants including CYC $(\mathrm{n}=7)$, AZA $(\mathrm{n}=5), \operatorname{CSA}(\mathrm{n}=3)$, tacrolimus $(n=4)$, methotrexate $(n=1)$, and mycophenolate mofetil $(n=1)$ were used for treating relapse in combination with glucocorticoids.

Patient survival and relapse. At 12 and 60 months, cumulative patient survival rates were $94.4 \%$ and $89.6 \%$, respectively (Figure 1A). During the observational period, 23 deaths were observed (7 infections, 3 vasculitis, 3 malignancies, 3 cardiovascular events, 1 asthma attack, 1 gastric ulcer, 1 suicide, 1 traffic accident, and 3 unknown cases) with median age of 68.0 years. Ten deaths occurred within 12 months of diagnosis. At 12 and 60 months, cumulative patient relapse-free survival rates assessed by Kaplan-Meier survival curve were $89.6 \%$ and $64.0 \%$, respectively (Figure 1B). At 12 and 60 months, cumulative patient relapse rates assessed by cumulative incidence function were $5.9 \%$ and $26.9 \%$, respectively (Figure 1C). During the observational period, 52 patients had 1 or more relapses. Median duration from diagnosis to the first relapse was 44.5 months, and median eosinophil count at the first relapse was $824 / \mu l$. Thirteen of 21 patients who were ANCA-positive at onset were ANCA-positive at the first relapse, while 4 of 31 patients who were ANCA-negative at onset became ANCA-positive at the first relapse. Organ involvement in the first relapses were nerve $(n=29)$; respiratory $(n=7)$; ear, nose, throat $(n=6)$; intestine $(n=5)$; skin $(n=4)$, and heart $(n=3)$. Thirty-one of 52 patients had major relapses.

Multivariate analysis for relapse. A total of 24 items were selected as potential explanatory variables: age, sex, MPO-/PR3-ANCA status, WBC count, eosinophil count, serum IgE, CRP and creatinine levels, 8 systems of organ involvement, FFS, initial dose of oral glucocorticoids, dose of oral glucocorticoids at 6 months, use of methylprednisolone pulse therapy, use of IVIG, and use of immunosuppressants in remission induction and maintenance phases. We confirmed in advance there was no collinearity between

Personal non-commercial use only. The Journal of Rheumatology Copyright $\subset$ 2018. All rights reserved. 

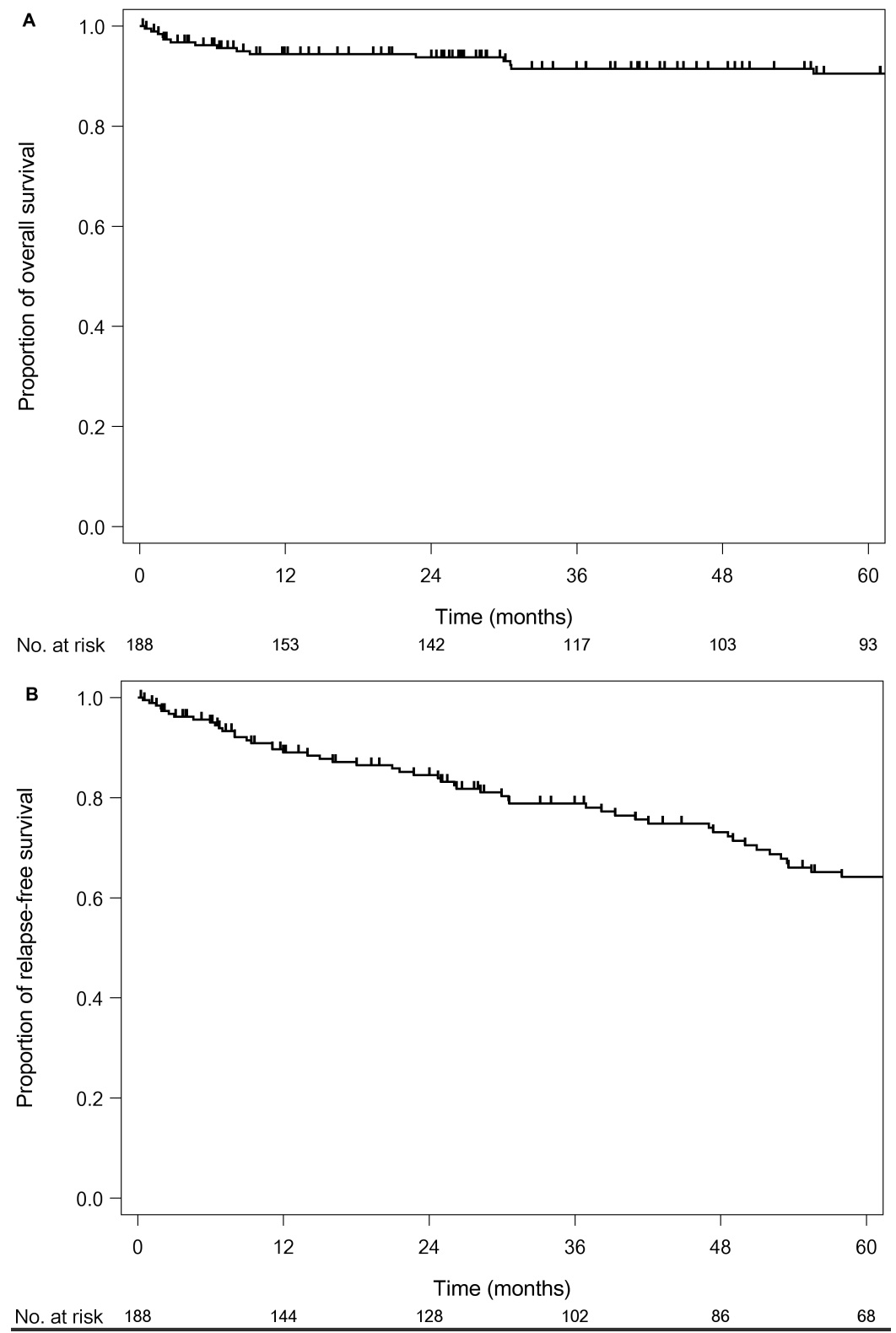

Figure 1. Patient survival, relapse-free survival and relapse rates. A. Cumulative survival rates assessed by Kaplan-Meier survival curve were $94.4 \%$ and $89.6 \%$ at 12 and 60 months, respectively. B. Cumulative relapse-free survival rates assessed by Kaplan-Meier survival curve were $89.6 \%$ and $64.0 \%$ at 12 and 60 months, respectively.

them. Explanatory variables with $\mathrm{p}$ values $<0.10$ on univariate analysis were entered in the multivariate analysis model.

Multivariate analyses were performed with 2 models, a proportional hazards model for relapse-free survival and a competing risk model for relapse (Table 3 ). In the proportional hazards model, significant factors for lower relapse risks were higher eosinophil count at onset and AZA maintenance therapy. The factor for higher risks was higher serum $\mathrm{IgE}$ level at onset. Relapse-free survival curves were significantly different between the CYC induction without AZA maintenance group and the CYC induction followed by AZA maintenance group ( $\mathrm{p}=0.03$, Figure $2 \mathrm{~A})$.

In the competing risk model, age, sex, and serum creatinine level were identified as statistically significant factors by univariate analysis. However, none of those factors were statistically significant in multivariate analysis.

Asthma exacerbation and EGPA relapse. During the observational period, 73 of 188 patients (38.8\%) had asthma exacerbation without or before EGPA relapse. Median duration from EGPA diagnosis to asthma exacerbation was 27 months, and median dose of glucocorticoids at exacer- 


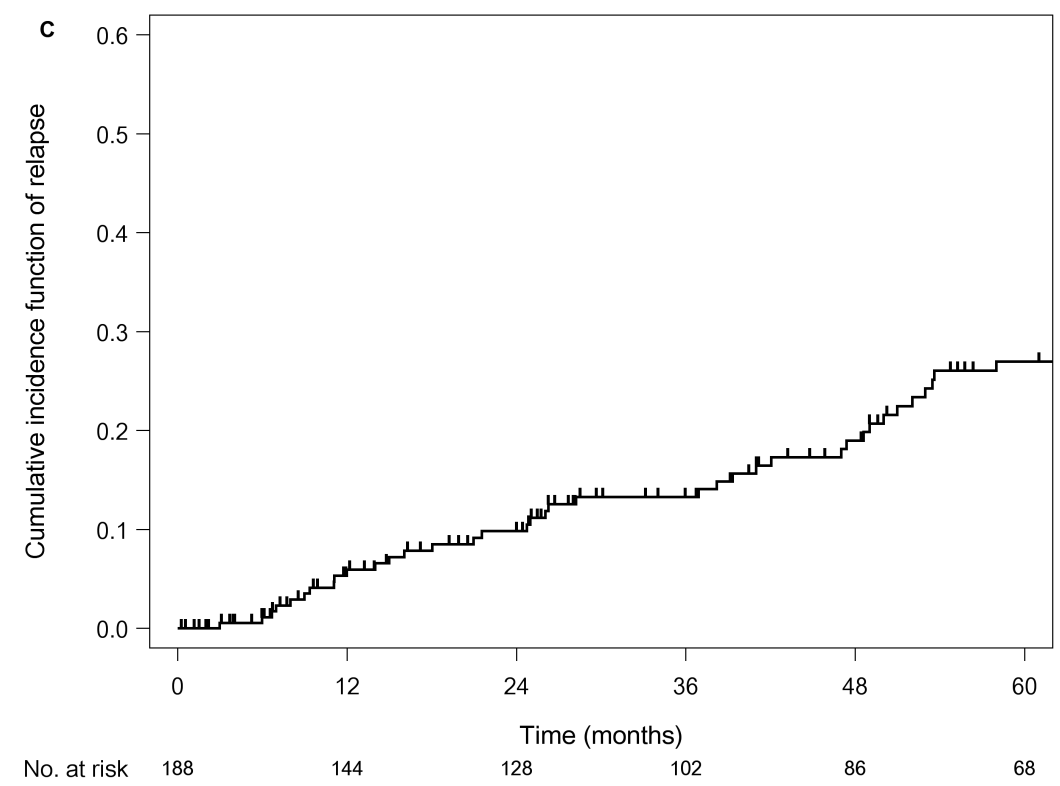

Figure 1. C. Cumulative relapse rates assessed by cumulative incidence function were $5.9 \%$ and $26.9 \%$ at 12 and 60 months, respectively.

Table 3. Multivariate analysis for relapse.

\begin{tabular}{|c|c|c|c|c|c|c|}
\hline \multirow[t]{2}{*}{ Variables } & \multicolumn{3}{|c|}{ Univariate Analysis } & \multicolumn{3}{|c|}{ Multivariate Analysis } \\
\hline & HR & $95 \% \mathrm{CI}$ & $\mathrm{p}$ & HR & $95 \% \mathrm{CI}$ & $\mathrm{p}$ \\
\hline \multicolumn{7}{|l|}{ Proportional hazards model } \\
\hline $\mathrm{IgE}, 100 \mathrm{IU} / \mathrm{ml}$ & 1.016 & $1.002-1.031$ & 0.029 & 1.025 & $1.011-1.039$ & $<0.001$ \\
\hline \multicolumn{7}{|l|}{ ANCA } \\
\hline Negative & Ref. & & & Ref. & & \\
\hline None & Ref. & & & Ref. & & \\
\hline CYC for induction & 1.746 & $1.023-2.981$ & 0.041 & 1.285 & $0.673-2.452$ & 0.447 \\
\hline $\begin{array}{l}\mathrm{CYC} \text { for induction }+\mathrm{AZA} \\
\text { for maintenance }\end{array}$ & 0.413 & $0.163-1.045$ & 0.062 & 0.261 & $0.079-0.870$ & 0.029 \\
\hline \multicolumn{7}{|l|}{ Competing risk model } \\
\hline Age & 0.984 & $0.966-1.002$ & 0.087 & 0.984 & $0.967-1.002$ & 0.076 \\
\hline
\end{tabular}

Eosino: eosinophil count; IgE: immunoglobulin E; ANCA: antineutrophil cytoplasmic antibodies; IS: immunosuppressant; CYC: cyclophosphamide; AZA: azathioprine.

bation was $7.0 \mathrm{mg} /$ day. On asthma exacerbation, some physicians considered it a sign of EGPA relapse, while others considered it an independent event of EGPA relapse. In this study, 21 of 73 patients received increased doses of systemic glucocorticoids at asthma exacerbation. The remaining 52 patients received the same dose of systemic glucocorticoids/immunosuppressants at asthma exacerbation. Relapse-free survival curves after asthma exacerbation were not significantly different between the 2 groups $(\mathrm{p}=0.60$, Figure 2B) .

\section{DISCUSSION}

In our study, we aimed to investigate factors associated with relapse in EGPA by analyzing a large and well-characterized Japanese cohort. Phenotypic features of our cohort, such as eosinophil count, ANCA positivity, and frequency of neuropathy, were mostly consistent with other large cohorts $^{9,10,11,14,17}$. A multicenter design and large sample size in our study reduced potential bias, and consequently our cohort reflected general patients with EGPA. Regarding the outcomes, the cumulative survival and relapse-free survival

$$
\text { Personal non-commercial use only. The Journal of Rheumatology Copyright } \subset \text { C 2018. All rights reserved. }
$$




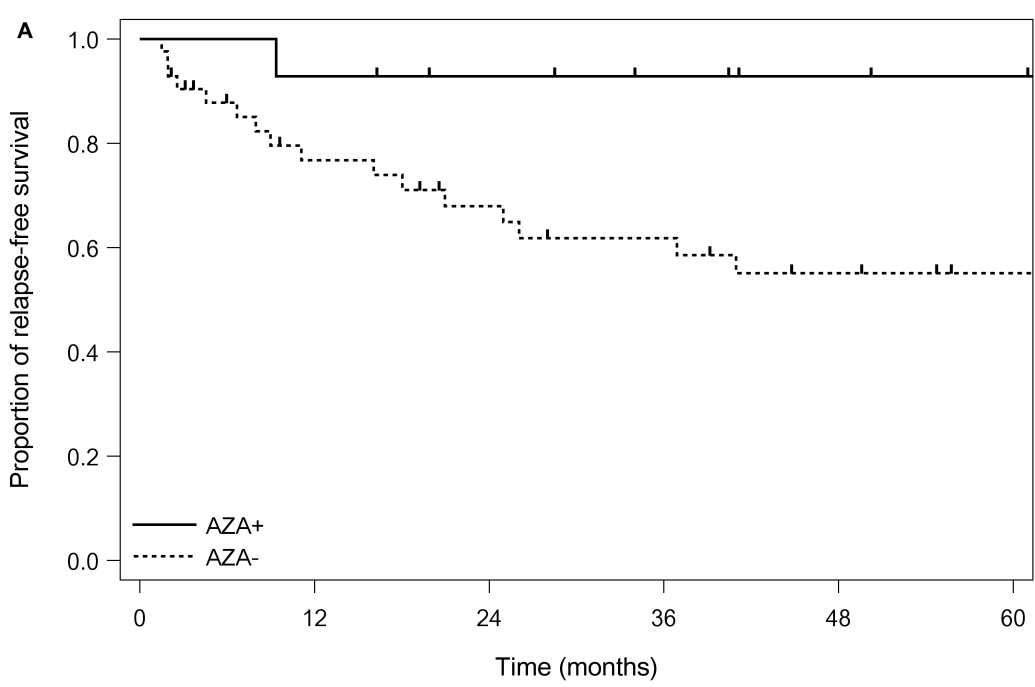

\begin{tabular}{ccccccc}
\multicolumn{2}{c}{ No. at risk } & & & & \\
AZA+ & 14 & 13 & 11 & 9 & 7 & 6 \\
AZA- & 42 & 27 & 22 & 19 & 15 & 12
\end{tabular}

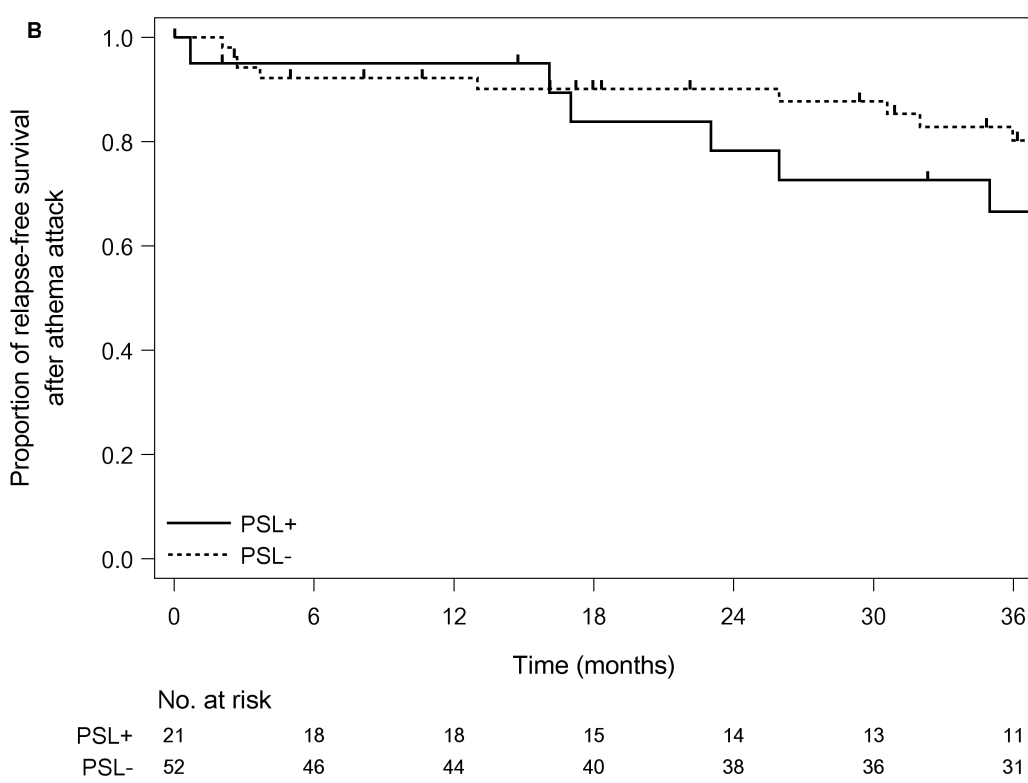

Figure 2. Relapse-free survival in the subgroups of the cohort. A. Of 56 patients who received cyclophosphamide in the remission induction phase, 14 patients were treated by AZA maintenance therapy. The cumulative relapse-free survival rate was lower in the patients treated with AZA maintenance therapy than the remaining 42 patients $(p=0.03)$. B. Of 73 patients who experienced asthma exacerbation without or before relapse, 21 patients received an increased dose of systemic PSL at exacerbation. The remaining 52 patients did not undergo changes in systemic treatments at the time. Cumulative relapse-free survival rate was not significantly different between the 2 groups $(\mathrm{p}=0.60)$. The starting point of the Kaplan-Meier curve was the time of asthma exacerbation. AZA: azathioprine; PSL: prednisolone.

rates at 5 years were $89.6 \%$ and $64.0 \%$, respectively. Other cohort studies reported 5-year cumulative survival rates of $88-97 \% 10,11,16,17$ and 5-year relapse-free survival rates of $54-58 \% 16,17$, also comparable with our data. Regarding the risk factors for relapse, we identified higher eosinophil count at onset and AZA maintenance therapy as independent factors, with lower risks and higher IgE levels at onset as a higher risk factor for relapse by multivariate analysis with a proportional hazards model. In the other cohort studies, multivariate analyses with a proportional hazards model were 
done, except in the study by Tsurikisawa, et al, which used logistic regression ${ }^{11}$. Durel, et al reported being Italian as an independent factor with a lower risk ${ }^{9}$. Both Samson, et al and Comarmond, et al reported higher eosinophil count as an independent factor with a lower risk ${ }^{16,17}$, which was common to our result. Tsurikisawa, et al reported GI tract and myocardial involvements as higher risk factors ${ }^{11}$. The reason for those differences was not clear, but differences in definitions of relapse and treatment strategies might have influenced the results.

In addition to the conventional proportional hazards model, we performed multivariate analysis with the competing risk model. The proportional hazards model for relapse-free survival evaluated a composite event of relapse and death, while the competing risk model for relapse evaluated only a relapse event. In our study, no risk factors for relapse were identified with the competing risk model. Regarding pure relapse risk, the risk factors identified by the proportional hazards model might be overestimated. Conversely, there is also a possibility that the smaller event number in the competing risk model made it difficult to identify significant factors.

The benefit of immunosuppressant maintenance therapy in preventing relapse of EGPA is still being debated. In our study, multivariate analysis with the proportional hazards model suggested the efficacy of AZA maintenance therapy, while it was not a statistically significant factor in the competing risk model. Among the other cohort studies, Durel, et al reported that being Italian was associated with lower relapse risk ${ }^{9}$. They speculated that longer-term glucocorticoid and immunosuppressant use in Italy might prevent relapse, which might support our results ${ }^{9}$. Recently, the French Vasculitis Study Group published the results of a randomized controlled trial (CHUSPAN2) ${ }^{24}$ for nonsevere vasculitides including EGPA $(\mathrm{n}=51)$, MPA $(\mathrm{n}=25)$, and polyarteritis nodosa $(n=19)$. Contrary to our results, the group reported that AZA was not superior to placebo in preventing relapse. However, the mixed subjects with different vasculitides in the trial decreased the certainty of conclusion for each vasculitis, and AZA was administered for only 12 months. The efficacy of longterm AZA maintenance therapy is still unclear. A carefully designed clinical trial for EGPA is required to clarify this issue. In any case, there is obviously an unmet need for preventing relapse of EGPA.

Eosinophil count is often parallel to serum IgE level. However, higher eosinophil count was associated with a lower risk for relapse, whereas higher serum IgE level was associated with a relapse risk. The reason for this dissociation was not clear. Patients with higher eosinophil count or serum $\mathrm{IgE}$ level at disease onset may tend to present higher eosinophil count or serum IgE level during disease course. Physicians generally attach more importance to eosinophil count than to serum IgE level in their decision making (e.g., speed of glucocorticoids tapering). That practice might be a cause of the dissociation.
Another issue in EGPA is the meaning of asthma exacerbation during followup. Asthma exacerbation has sometimes been considered a symptom of active EGPA or a sign for high risk of EGPA relapse. In fact, "wheeze" was included in BVAS, a standard assessment tool for disease activity of $\mathrm{AAV}^{22,23}$. On the other hand, allergic airway inflammation in asthma is a different condition from granulomatous vasculitis in EGPA. Therefore, there has also been an opinion that asthma exacerbation is an independent event from vasculitis. This leads to a question of whether systemic treatment change for EGPA is needed with asthma exacerbation during EGPA followup. Of 73 patients having asthma exacerbation in our cohort, 21 received an increased dose of systemic glucocorticoids, while the remaining 52 did not. Frequencies of EGPA relapse following asthma exacerbation were not significantly different between the 2 groups. Our result suggests that systemic treatment change for EGPA is not necessary in such patients.

There are limitations in our study. The design of the retrospective observational study prevents clear conclusions about the treatments. Treatments obviously influence the outcomes and could be confounding factors in the nontrial setting. For example, CYC has been used more frequently for patients with a positive ANCA test, renal involvement, and/or higher FFS. Next, we did not assess the detail of tapering dose of glucocorticoids. In our study, the dose of glucocorticoids at onset, 6,12 , and 36 months was assessed. Different tapering speeds of glucocorticoid dose might have influenced EGPA relapse, although the dose of glucocorticoids was not significantly different at all the timepoints between ANCA-positive and ANCA-negative groups, or between glucocorticoid monotherapy and CYC combination therapy groups (data not shown).

We retrospectively analyzed factors associated with relapse in patients with EGPA using the 2 multivariate analysis models. The potential benefit of AZA maintenance therapy in preventing relapse of EGPA was suggested by the proportional hazards model. However, because there was a discrepancy in the results between the models, we could not reach a clear conclusion. Future studies are required to determine the efficacy of AZA maintenance therapy. The proportional hazards model also identified higher eosinophil counts at onset as an independent factor with a lower risk and higher IgE levels at onset as a higher risk factor for relapse in patients with EGPA.

\section{ACKNOWLEDGMENT}

The authors acknowledge Drs. Mieko Yamagata, Daisuke Kashiwakuma, and Takayoshi Owada for their contribution in data collection. The authors also acknowledge Dr. Kengo Nagashima for his statistical advice.

\section{REFERENCES}

1. Churg J, Strauss L. Allergic granulomatosis, allergic angiitis, and periarteritis nodosa. Am J Pathol 1951;27:277-301.

2. Jennette JC, Falk RJ, Bacon PA, Basu N, Cid MC, Ferrario F, et al.

Personal non-commercial use only. The Journal of Rheumatology Copyright (C) 2018. All rights reserved. 
2012 revised international Chapel Hill consensus conference nomenclature of vasculitides. Arthritis Rheum 2013;65:1-11.

3. Sada KE, Amano K, Uehara R, Yamamura M, Arimura Y, Nakamura $\mathrm{Y}$, et al. A nationwide survey on the epidemiology and clinical features of eosinophilic granulomatosis with polyangiitis (Churg Strauss) in Japan. Mod Rheumatol 2014;24:640-4.

4. Fujimoto S, Watts RA, Kobayashi S, Suzuki K, Jayne DR, Scott DG, et al. Comparison of the epidemiology of anti-neutrophil cytoplasmic antibody-associated vasculitis between Japan and the U.K. Rheumatology 2011;50:1916-20

5. Schleimer RP, Bochner BS. The effects of glucocorticoids on human eosinophils. J Allergy Clin Immunol 1994;94:1202-13.

6. Pagnoux C, Guilpain P, Guillevin L. Churg-Strauss syndrome. Curr Opin Rheumatol 2007;19:25-32.

7. Ribi C, Cohen P, Pagnoux C, Mahr A, Arène JP, Lauque D, et al. Treatment of Churg-Strauss syndrome without poor-prognosis factors: a multicenter, prospective, randomized, open-label study of seventy-two patients. Arthritis Rheum 2008;58:586-94

8. Gayraud M, Guillevin L, Cohen P, Lhote F, Cacoub P, Deblois P, et al. Treatment of good-prognosis polyarteritis nodosa and Churg-Strauss syndrome: comparison of steroids and oral or pulse cyclophosphamide in 25 patients. French Cooperative Study Group for Vasculitides. Br J Rheumatol 1997;36:1290-7.

9. Durel CA, Berthiller J, Caboni S, Jayne D, Ninet J, Hot A. Long-term follow-up of a multicentre cohort of 101 patients with eosinophilic granulomatosis with polyangiitis (EGPA). Arthritis Care Res 2016;68:374-87.

10. Moosig F, Bremer JP, Hellmich B, Holle JU, Holl-Ulrich K, Laudien $\mathrm{M}$, et al. A vasculitis centre based management strategy leads to improved outcome in eosinophilic granulomatosis and polyangiitis (Churg-Strauss, EGPA): monocentric experiences in 150 patients. Ann Rheum Dis 2013;72:1011-7.

11. Tsurikisawa N, Oshikata C, Kinoshita A, Tsuburai T, Saito H. Longterm prognosis of 121 patients with eosinophilic granulomatosis with polyangiitis in Japan. J Rheumatol 2017;44:1206-15.

12. Robson J, Doll H, Suppiah R, Flossmann O, Harper L, Höglund P, et al. Glucocorticoid treatment and damage in the anti-neutrophil cytoplasm antibody-associated vasculitides: long-term data from the European Vasculitis Study Group trials. Rheumatology 2015;54:471-81.

13. Guillevin L, Lhote F, Gayraud M, Cohen P, Jarrousse B, Lortholary $\mathrm{O}$, et al. Prognostic factors in polyarteritis nodosa and Churg-Strauss syndrome. A prospective study in 342 patients. Medicine 1996;75:17-28.
14. Guillevin L, Pagnoux C, Seror R, Mahr A, Mouthon L, Le Toumelin P. The Five-Factor Score revisited: assessment of prognoses of systemic necrotizing vasculitides based on the French Vasculitis Study Group (FVSG) cohort. Medicine 2011;90:19-27.

15. Cohen P, Pagnoux C, Mahr A, Arène JP, Mouthon L, Le Guern V, et al. Churg-Strauss syndrome with poor-prognosis factors: a prospective multicenter trial comparing glucocorticoids and six or twelve cyclophosphamide pulses in forty-eight patients. Arthritis Rheum 2007;57:686-93.

16. Samson M, Puéchal X, Devilliers H, Ribi C, Cohen P, Stern M, et al. Long-term outcomes of 118 patients with eosinophilic granulomatosis with polyangiitis (Churg-Strauss syndrome) enrolled in two prospective trials. J Autoimmun 2013;43:60-9.

17. Comarmond C, Pagnoux C, Khellaf M, Cordier JF, Hamidou M, Viallard JF, et al. Eosinophilic granulomatosis with polyangiitis (Churg-Strauss): clinical characteristics and long-term followup of the 383 patients enrolled in the French Vasculitis Study Group cohort. Arthritis Rheum 2013;65:270-81.

18. Ntatsaki E, Carruthers D, Chakravarty K, D'Cruz D, Harper L, Jayne D, et al. BSR and BHPR guideline for the management of adults with ANCA-associated vasculitis. Rheumatology 2014;53:2306-9.

19. Yates M, Watts RA, Bajema IM, Cid M, Crestani B, Hauser T, et al. EULAR/ERA-EDTA recommendations for the management of ANCA-associated vasculitis. Ann Rheum Dis 2016;75:1583-94.

20. Masi AT, Hunder GG, Lie JT, Michel BA, Bloch DA, Arend WP, et al. The American College of Rheumatology 1990 criteria for the classification of Churg-Strauss syndrome (allergic granulomatosis and angiitis). Arthritis Rheum 1990;33:1094-100.

21. Lanham JG, Elkon KB, Pusey CD, Hughes GR. Systemic vasculitis with asthma and eosinophilia: a clinical approach to the Churg-Strauss syndrome. Medicine 1984;63:65-81.

22. Luqmani RA, Bacon PA, Moots RJ, Janssen BA, Pall A, Emery P, et al. Birmingham Vasculitis Activity Score (BVAS) in systemic necrotizing vasculitis. QJM 1994;87:671-8.

23. Mukhtyar C, Lee R, Brown D, Carruthers D, Dasgupta B, Dubey S, et al. Modification and validation of the Birmingham Vasculitis Activity Score (version 3). Ann Rheum Dis 2009;68:1827-32.

24. Puéchal X, Pagnoux C, Baron G, Quémeneur T, Néel A, Agard C, et al. Adding azathioprine to remission-induction glucocorticoids for eosinophilic granulomatosis with polyangiitis (Churg-Strauss), microscopic polyangiitis, or polyarteritis nodosa without poor prognosis factors: A randomized, controlled trial. Arthritis Rheumatol 2017;69:2175-86. 